\title{
Differential expression of RecQ-like helicase 4 in triple negative breast cancer.
}

Shahan Mamoor, MS ${ }^{1}$

$3 \quad$ shahanmamoor@gmail.com East Islip, NY 11730

Women diagnosed with triple negative breast cancer can benefit neither from endocrine therapy nor from HER2-targeted therapies ${ }^{1}$. We mined published microarray datasets ${ }^{2,3}$ to determine in an unbiased fashion and at the systems level genes most differentially expressed in the primary tumors of patients with breast cancer. We report here significant differential expression of the gene encoding RecQ-like helicase 4, RECQL4, when comparing the tumor cells of patients with triple negative breast cancer to normal mammary ductal cells ${ }^{2}$. RECQL4 was also differentially expressed in bulk tumor in human breast cancer ${ }^{3}$. RECQL4 mRNA was present at significantly increased quantities in TNBC tumor cells relative to normal mammary ductal cells. Analysis of human survival data revealed that expression of RECQL4 in primary tumors of the breast was correlated with recurrence-free survival in patients with HER2+ type cancer, while within triple negative breast cancer, primary tumor expression of RECQL4 was correlated with distant metastasis-free survival in patients with basal-like 2 subtype disease. RECQL4 may be of relevance to initiation, maintenance or progression of triple negative breast cancers.

Keywords: breast cancer, RECQL4, RecQ-like helicase 4, systems biology of breast cancer, targeted therapeutics in breast cancer. 
Patients with hormone receptor positive disease can benefit from endocrine therapy ${ }^{4}$ : aromatase inhibition such as that provided by exemestane or letrozole, or estrogen receptor antagonism such as that provided by tamoxifen or fulvestrant. Patients whose tumors overexpress the human epidermal growth factor 2 (HER2) can be treated with HER2-targeted therapies: HER2 inhibitors, immunoglobulin ${ }^{5}$ - or small molecule-based ${ }^{6}$. As triple negative breast cancer (TNBC) is defined by its lack of HER2 expression as well as by its lack of expression of the hormone receptor estrogen, patients diagnosed with triple negative breast cancer will not benefit from either of these treatment strategies.

Understanding the transcriptional composition of triple negative breast cancers in humans can facilitate development of novel therapeutics and contribute to efforts to prevent progression to metastatic stages. We mined published microarray data ${ }^{2,3}$ to understand at the transcriptome level and in an unbiased fashion genes most differentially expressed in the tumor cells of patients with triple negative breast cancer.

\section{Methods}

We utilized datasets GSE38959 ${ }^{2}$ and GSE109169³ for this differential gene expression analysis of female breast cancer in conjunction with GEO2R. GSE38959 was generated using Agilent-014850 Whole Human Genome Microarray 4x44K G4112F technology with $n=13$ samples of normal mammary gland ductal cells and $n=30$ samples of tumor cells from patients with triple negative breast cancer; analysis was performed using platform GPL4133. GSE109169 was generated using Affymetrix Human Exon 1.0 ST Array technology with $n=25$ normal breast tissue and $n=25$ tumors of the breast; analysis was performed using platform GPL5175. The tissues from this dataset are paired tissues ( 25 tumors matching 25 normal breast tissues from 25 patients). The Benjamini and Hochberg method of $p$-value adjustment was used for ranking of differential expression but raw $p$-values were used to assess statistical significance of global differential expression. Log-transformation of data was auto-detected, and the NCBI-generated category of platform annotation was used. A statistical test was performed to evaluate whether RECQL4 expression was significantly different between primary breast tumors and breast tissue using a two-tailed t-test.

For Kaplan-Meier survival analysis, we used the Kaplan-Meier plotter tool ${ }^{7}$ for correlation of RECQL4 mRNA expression levels with recurrence-free survival (RFS) in $n=296$ patients with basal subtype cancer, $n=222$ patients with luminal A subtype cancer, $n=200$ patients with luminal B subtype cancer, $n=198$ patients with HER $2+$ cancer, and $n=27$ patients with normal-like subtype cancer. In patients with triple negative breast cancer $^{7}$, we analyzed distant metastasis-free survival (DMFS) for $n=76$ patients with basal-like 1 subtype TNBC, $n=54$ patients with basal-like 2 subtype TNBC, $n=93$ patients with immunomodulatory subtype TNBC, $n=64$ patients with luminal androgen receptor subtype TNBC, $n=73$ patients with mesenchymal subtype TNBC, and $n=29$ patients with mesenchymal stem-like subtype TNBC.

\section{Results}

We performed discovery of genes associated with triple negative breast cancer in humans by mining two independently published microarray datasets ${ }^{2,3}$.

\section{RECQL4 is differentially expressed in primary tumors and tumor cells of the breast.}

Comparison of the tumor cells of patients with triple negative breast cancer to normal mammary ductal cells ${ }^{2}$ revealed RecQ-like helicase 4, encoded by RECQL4, as among the genes whose expression was most significantly different in human TNBC as compared to the normal breast (Chart 1$)$. When sorting each of the genes expressed in the tumor cells of patients with triple negative breast cancer based on significance of difference as compared to normal mammary ductal cells, RECQL4 ranked 247 out of 45015 total transcripts, equating to $99.5 \%$ differential expression (Chart 1). Differential expression of RECQL4 in the tumor cells of patients with triple negative breast cancer was statistically significant 


\section{RECQL4 is expressed at significantly higher levels in triple negative tumor cells as compared to normal cells of the breast.}
We obtained exact mRNA expression levels for RECQL4 - from normal mammary ductal cells of the breast and from tumor cells of patients with triple negative breast cancer - to understand the magnitude and direction of RECQL4 expression change in TNBC. RECQL4 mRNA transcript was present at higher levels in triple negative tumor cells of the breast as compared to normal mammary ductal cells (Figure 1). Increased expression of RECQL4 in primary tumor-isolated triple negative breast cancer cells was statistically significant (Figure 1: $p<0.0001$ ). RECQL4 was expressed at $18.9 \pm 8.3$ arbitrary units (A.U.) in normal mammary ductal cells, while it was expressed at $71.6 \pm 36.2$ A.U. in tumor cells of patients with triple negative breast cancer. We calculated a mean fold change of 3.8 when comparing triple negative breast cancer cells to normal mammary ductal cells.

\section{RECQL4 expression correlates with survival outcomes in HER2+ human breast cancer.}
We performed Kaplan-Meier survival analysis to evaluate potential relationships between primary tumor RECQL4 mRNA expression levels and survival outcomes in patients with breast cancer, when analyzing outcomes based on the molecular subtype of the patient (PAM50 classification): luminal A, luminal B, HER2+, normal-like, and basal-like. We found a statistically significant correlation between RECQL4 expression and recurrence-free survival (RFS) in patients with HER2+ subtype breast cancer (Figure 2; log rank $p$-value: 0.028 for recurrence-free survival, hazard ratio: 1.88 (1.06-3.32) (Fig. 2)).
RECQL4 mRNA levels were a negative prognostic indicator in HER2+ breast cancer patients. RFS was, on average, markedly greater for HER2+ patients with low tumor expression of RECQL4 as compared to HER2+ patients with high tumor expression of RECQL4 (Chart 3).
RECQL4 primary tumor expression was not correlated with recurrence-free survival in basal subtype (Figure 2; log rank $p$-value: 1 for RFS, hazard ratio: 1 (0.63-1.59) (Fig. 2), luminal A subtype (Figure 2; log rank $p$-value: 0.28 for RFS, hazard ratio: 1.49 (0.72-3.07) (Fig. 2)), in luminal B breast cancer (Figure 2; log rank $p$-value: 0.36 for RFS, hazard ratio: $1.3(0.74-2.29)$ (Fig. 2)), or in the normal-like subtype (Figure 2; log rank $p$-value: 0.34 for RFS, hazard ratio: 0.52 (0.13-2.07) (Fig. 2)).

\section{Influence of primary tumor RECQL4 expression on survival outcomes based on TNBC molecular} subtype.

We also performed Kaplan-Meier survival analysis to examine relationships between RECQL4 
mRNA expression levels with survival strictly in patients with triple negative breast cancer, analyzing outcomes based on the TNBC molecular subtype of the patient: basal-like 1, basal-like 2, immunomodulatory, mesenchymal, mesenchymal stem-like, and luminal androgen receptor. We observed a correlation between RECQL4 expression and distant metastasis-free survival (DMFS) in TNBC patients with basal-like 2 subtype disease which trended towards statistical significance (Figure 3; log rank $p$-value: 0.082 for distant metastasis-free survival, hazard ratio: 2.13 (0.89-5.11) (Fig. 3)). RECQL4 mRNA levels were a negative prognostic indicator in patients with basal-like 2 TNBC. Median DMFS was 35.24 months for basal-like 2 TNBC patients with low tumor expression of RECQL4 while median DMFS was 17.28 months for basal-like 2 TNBC patients with high tumor expression of RECQL4 (Chart 4).

RECQL4 primary tumor expression was not correlated with distant metastasis-free survival in basal-like 1 subtype (Figure 3; log rank $p$-value: 0.14 for DMFS, hazard ratio: 1.99 (0.79-4.99) (Fig. 3), immunomodulatory subtype (Figure 3; log rank $p$-value: 0.67 for DMFS, hazard ratio: $0.79(0.27-2.36)$ (Fig. 3)), in the mesenchymal subtype (Figure 3; log rank $p$-value: 0.87 for DMFS, hazard ratio: 1.07 (0.48-2.37) (Fig. 3)), in the mesenchymal stem-like subtype (Figure 3; log rank $p$-value: 0.29 for DMFS, hazard ratio: 1.82 (0.59-5.59) (Fig. 3)), or in the luminal androgen receptor subtype (Figure 3; log rank p-value: 0.52 for DMFS, hazard ratio: 1.33 (0.56-3.18) (Fig. 3)).

\section{Discussion}

We performed comparative transcriptome analysis using two independently published microarray datasets $^{2,3}$ to define and catalog transcriptional differences in human triple negative breast cancer. We provided evidence here that RecQ-like helicase 4, encoded by the RECQL4 gene, is a defining transcriptional feature of triple negative breast cancer. RECQL4 was also differentially expressed in the bulk tumors of patients with early-onset breast cancer. Expression of RECQL4 was correlated with recurrence-free survival in patients with HER2+ subtype breast cancer, and within triple negative breast cancer patients, primary tumor expression of RECQL4 was correlated with distant metastasis-free survival in patients with basal-like 2 subtype disease. RecQ-like helicase 4 and the molecular processes to which it pertains to may be relevant to the initiation or progression of triple negative breast cancer in humans. 


\section{References}

1. Foulkes, W.D., Smith, I.E. and Reis-Filho, J.S., 2010. Triple-negative breast cancer. New England journal of medicine, 363(20), pp.1938-1948.

2. Komatsu, M., Yoshimaru, T., Matsuo, T., Kiyotani, K., Miyoshi, Y., Tanahashi, T., Rokutan, K., Yamaguchi, R., Saito, A., Imoto, S. and Miyano, S., 2013. Molecular features of triple negative breast cancer cells by genome-wide gene expression profiling analysis. International journal of oncology, 42(2), pp.478-506.

3. Chang, J.W., Kuo, W.H., Lin, C.M., Chen, W.L., Chan, S.H., Chiu, M.F., Chang, I.S., Jiang, S.S., Tsai, F.Y., Chen, C.H. and Huang, P.H., 2018. Wild-type p53 upregulates an early onset breast cancer-associated gene GAS7 to suppress metastasis via GAS7-CYFIP1- mediated signaling pathway. Oncogene, 37(30), pp.4137-4150.

4. Spring, L.M., Gupta, A., Reynolds, K.L., Gadd, M.A., Ellisen, L.W., Isakoff, S.J., Moy, B. and Bardia, A., 2016. Neoadjuvant endocrine therapy for estrogen receptor-positive breast cancer: a systematic review and meta-analysis. JAMA oncology, 2(11), pp.1477-1486.

5. Slamon, D., Eiermann, W., Robert, N., Pienkowski, T., Martin, M., Press, M., Mackey, J., Glaspy, J., Chan, A., Pawlicki, M. and Pinter, T., 2011. Adjuvant trastuzumab in HER2-positive breast cancer. New England journal of medicine, 365(14), pp.1273-1283.

6. Park, J.W., Liu, M.C., Yee, D., Yau, C., van’t Veer, L.J., Symmans, W.F., Paoloni, M., Perlmutter, J., Hylton, N.M., Hogarth, M. and DeMichele, A., 2016. Adaptive randomization of neratinib in early breast cancer. New England Journal of Medicine, 375(1), pp.11-22.

7. Györffy, B., Lanczky, A., Eklund, A.C., Denkert, C., Budczies, J., Li, Q. and Szallasi, Z., 2010. An online survival analysis tool to rapidly assess the effect of 22,277 genes on breast cancer prognosis using microarray data of 1,809 patients. Breast cancer research and treatment, 123(3), pp.725-731. 


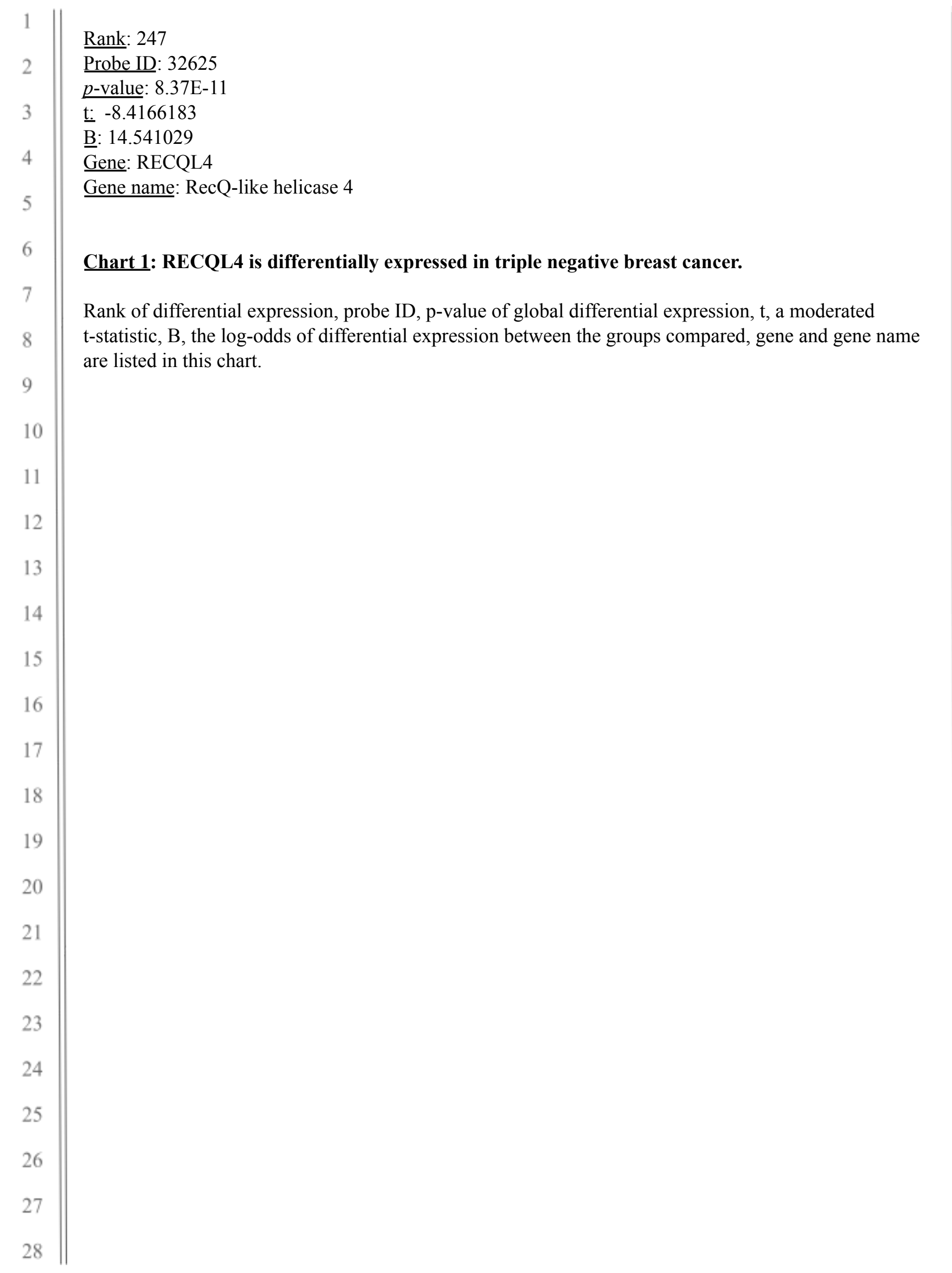




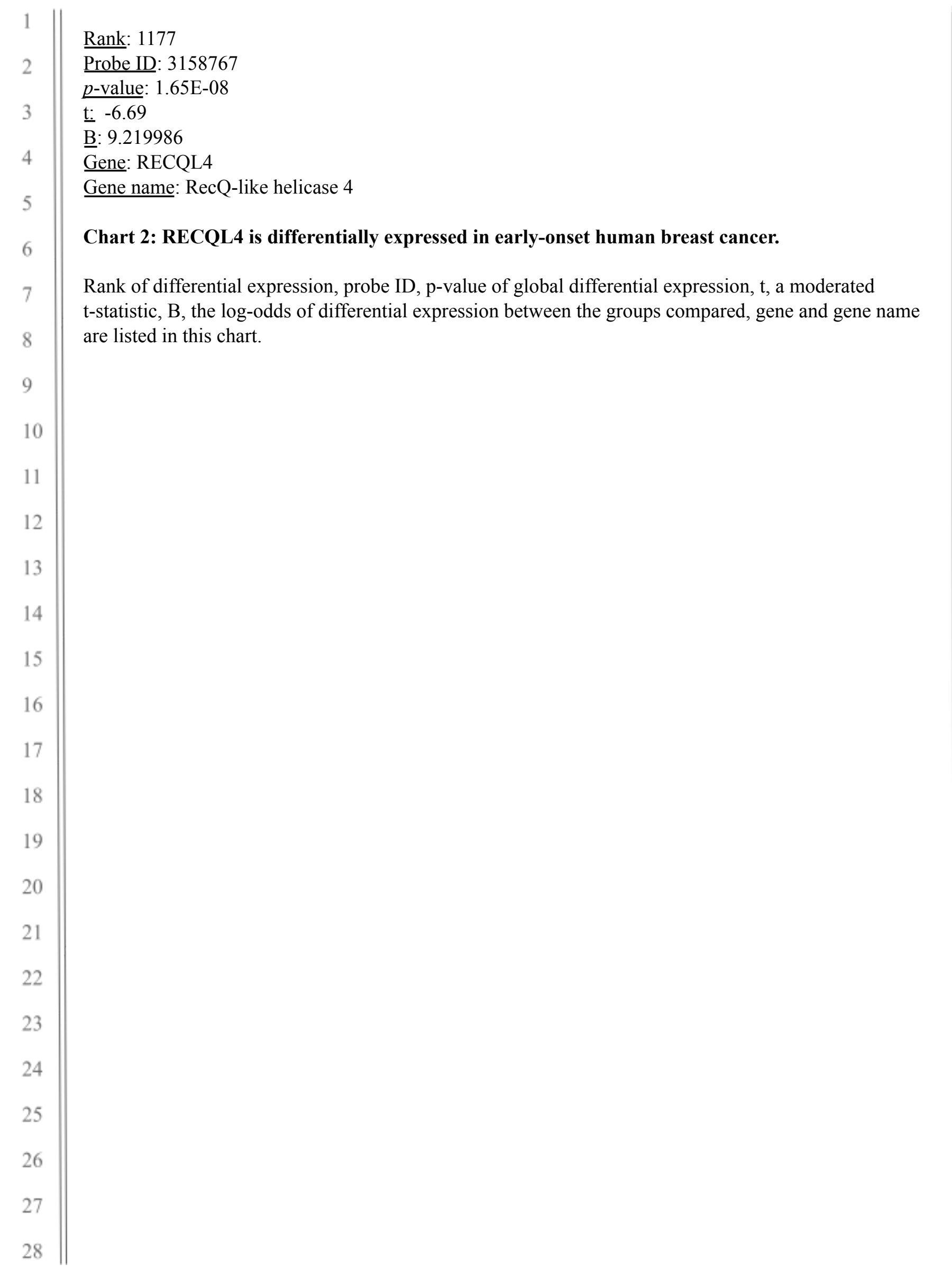




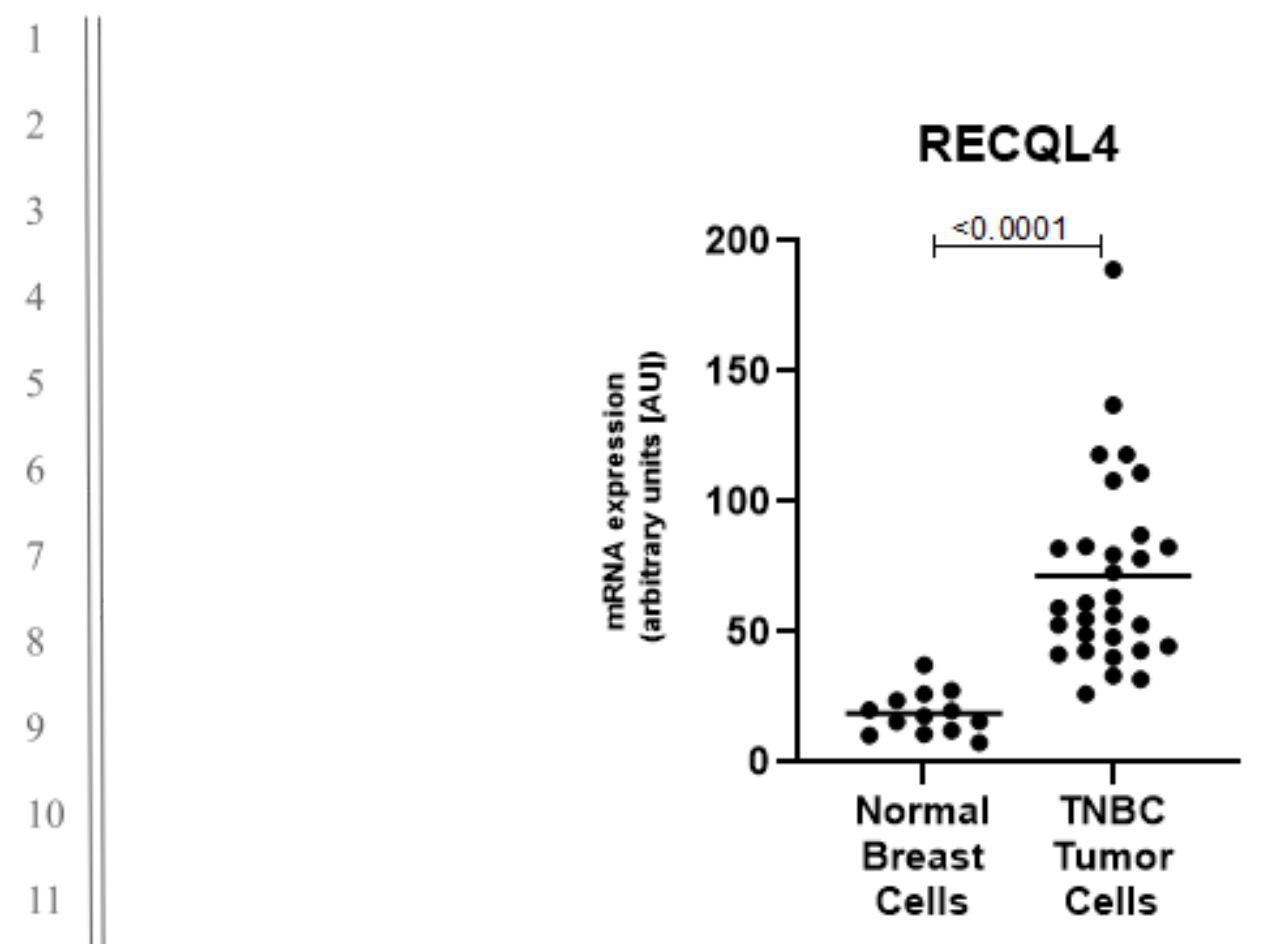

Figure 1: RECQL4 is expressed at significantly higher levels in primary TNBC tumor cells as compared to normal cells of the breast.

The mRNA expression level of RECQL4 in normal mammary ductal cells (left) and in the isolated primary tumor cells of patients with triple negative breast cancer (right) is graphically depicted with the result of a statistical test evaluating significance of difference in RECQL4 expression between normal cells of the breast and TNBC tumor cells, a $p$-value, listed above. 


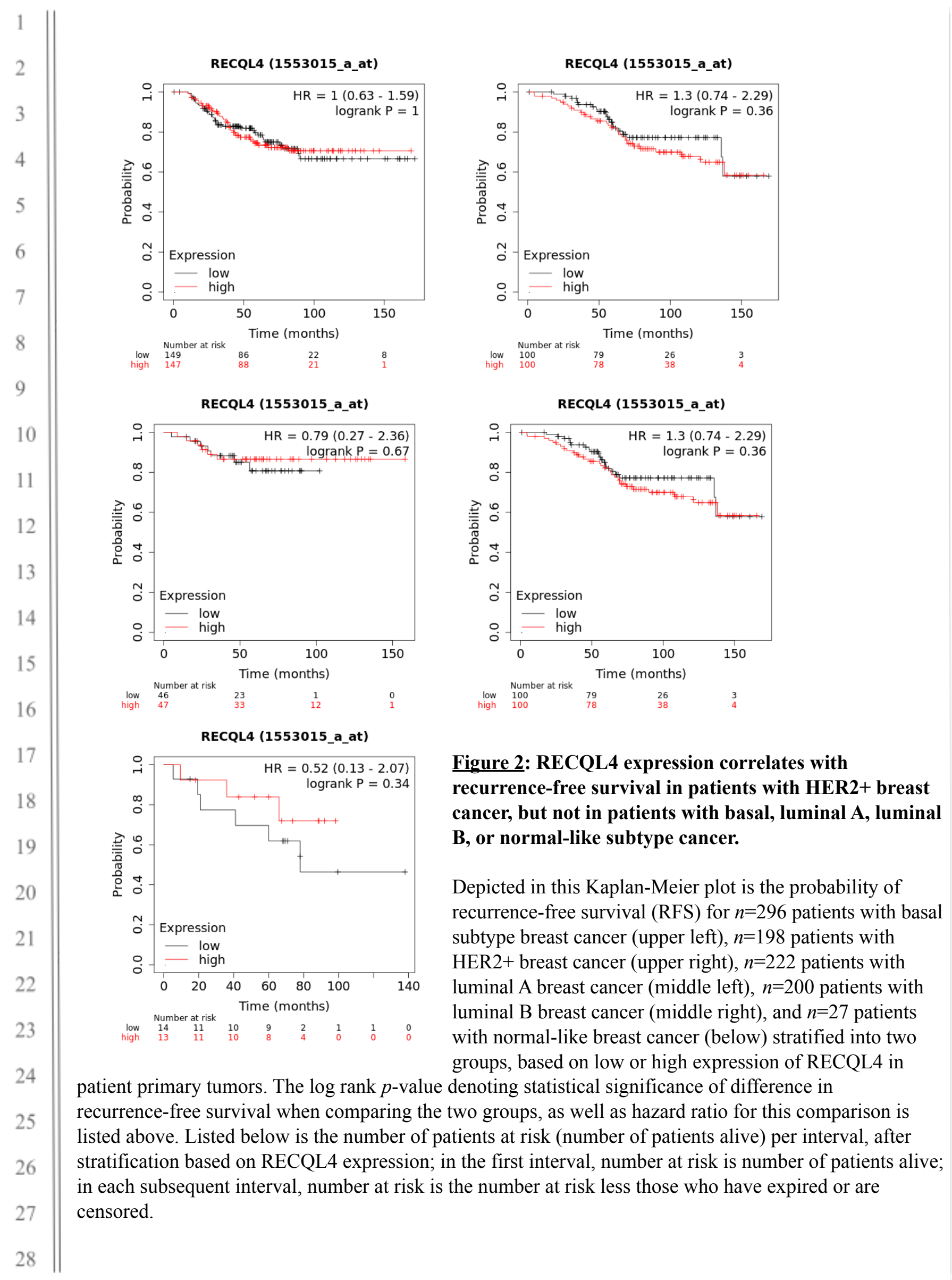



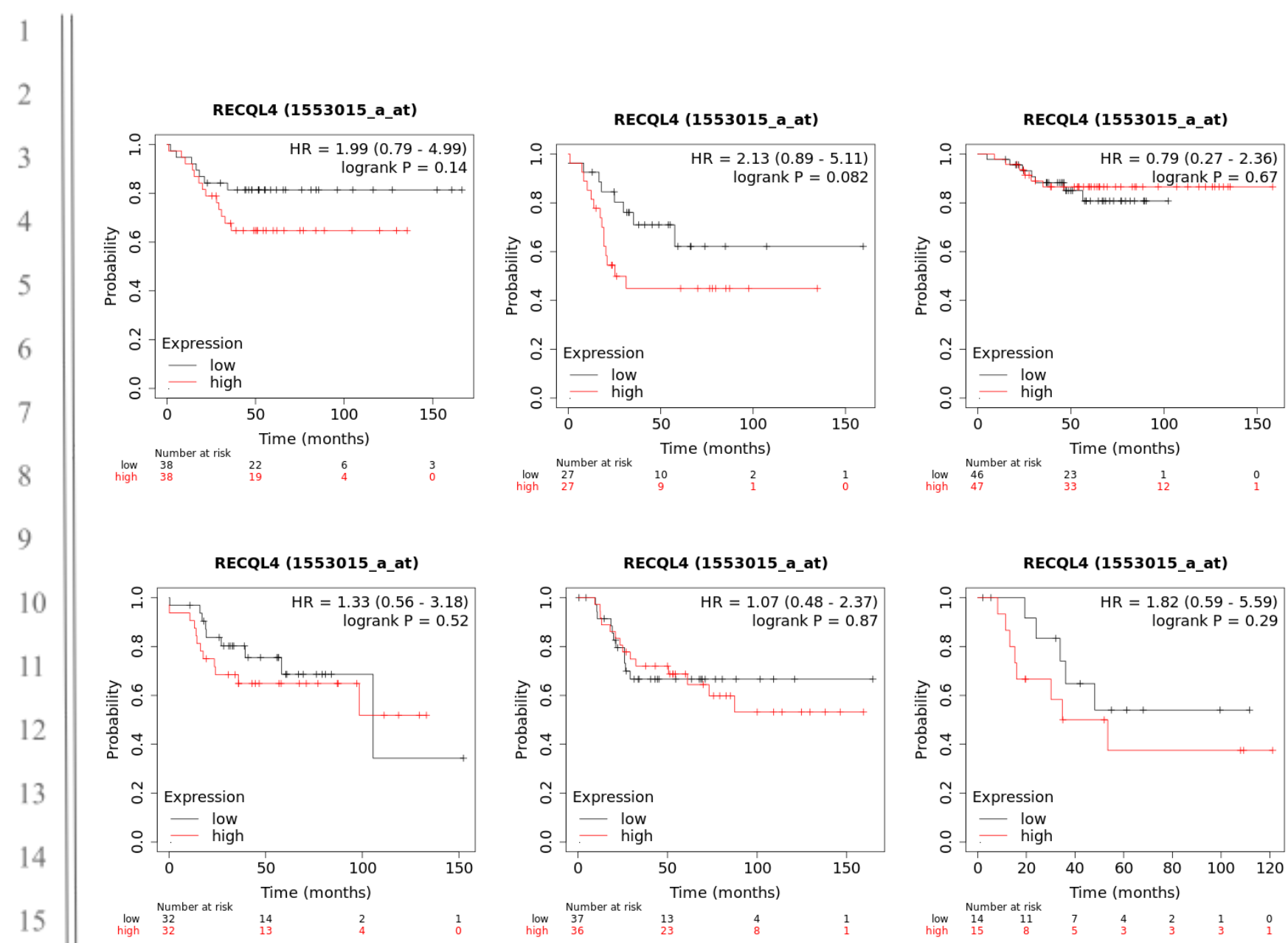

Figure 3: RECQL4 expression correlates with distant metastasis-free survival in triple negative breast cancer patients whose tumors are of the basal-like 2 molecular subtype.

Depicted in this Kaplan-Meier plot is the probability of distant metastasis-free survival (DMFS) for $n=76$ patients with basal-like 1 subtype TNBC (upper left), $n=54$ patients with basal-like 2 subtype TNBC (upper middle), $n=93$ patients with immunomodulatory subtype TNBC (upper right), $n=64$ patients with luminal androgen receptor subtype TNBC (lower left), $n=73$ patients with mesenchymal subtype TNBC (lower middle), and $n=29$ patients with mesenchymal stem-like subtype TNBC (lower right), stratified into two groups, based on low or high expression of RECQL4 in the primary tumors of patients with TNBC. The log rank p-value denoting statistical significance of difference in distant metastasis-free survival when comparing the two groups, as well as hazard ratio for this comparison is listed above. Listed below is the number of patients at risk (number of patients alive) per interval, after stratification based on RECQL4 expression; in the first interval, number at risk is number of patients alive; in each subsequent interval, number at risk is the number at risk less those who have expired or are censored. 


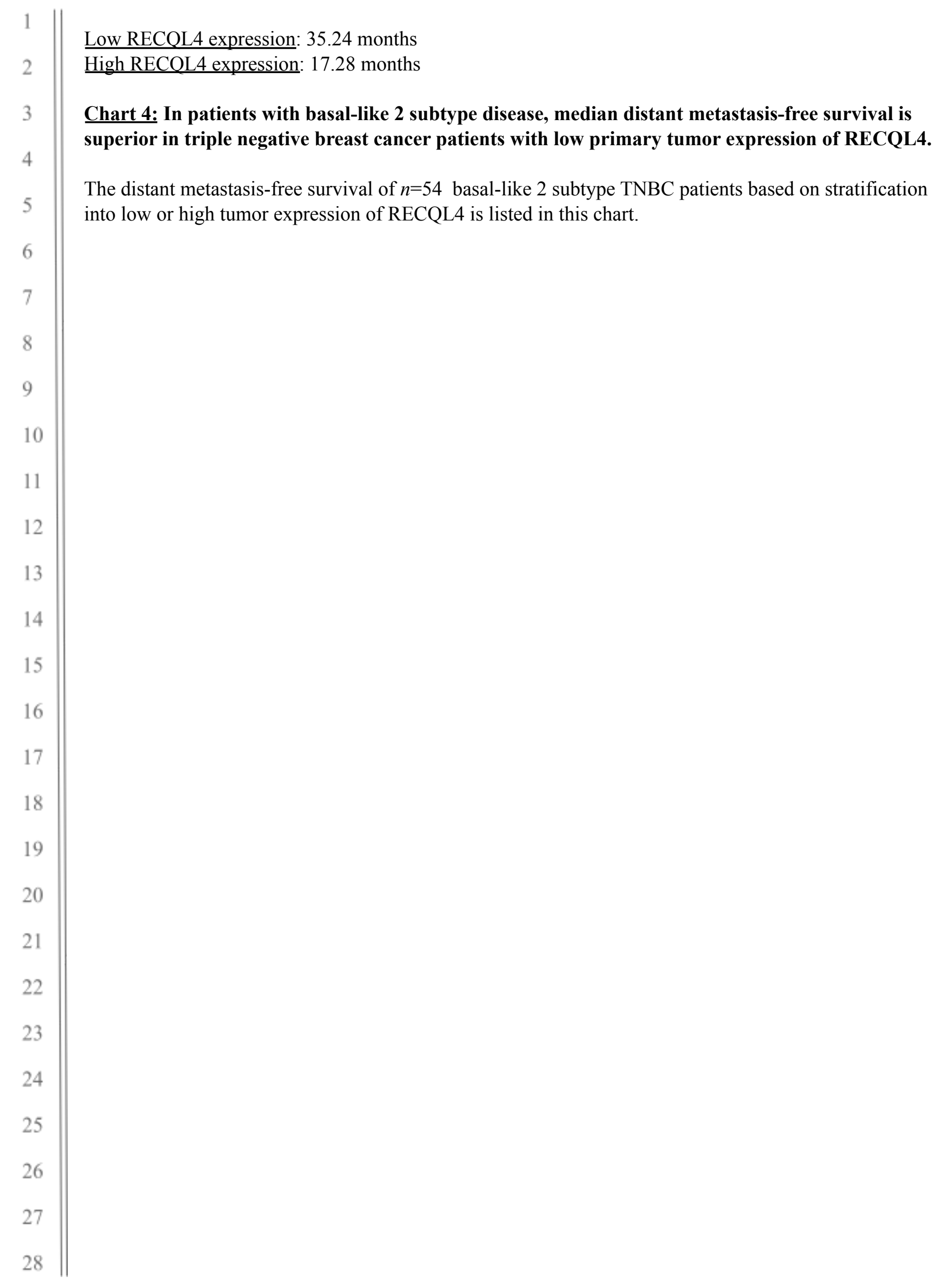

